University of Nebraska - Lincoln

DigitalCommons@University of Nebraska - Lincoln

U.S. Navy Research

U.S. Department of Defense

2011

Development of dengue DNA vaccines

Janine R. Danko

Infectious Diseases Directorate, Janine.danko@med.navy.mil

Charmagne G. Beckett

Uniformed Services University of the Health Sciences

Kevin R. Porter

Infectious Diseases Directorate

Follow this and additional works at: https://digitalcommons.unl.edu/usnavyresearch

Danko, Janine R.; Beckett, Charmagne G.; and Porter, Kevin R., "Development of dengue DNA vaccines" (2011). U.S. Navy Research. 31.

https://digitalcommons.unl.edu/usnavyresearch/31

This Article is brought to you for free and open access by the U.S. Department of Defense at DigitalCommons@University of Nebraska - Lincoln. It has been accepted for inclusion in U.S. Navy Research by an authorized administrator of DigitalCommons@University of Nebraska - Lincoln. 


\title{
Development of dengue DNA vaccines
}

\author{
Janine R. Danko ${ }^{\mathrm{a}, *}$, Charmagne G. Beckett ${ }^{\mathrm{b}, \mathrm{a}}$, Kevin R. Porter ${ }^{\mathrm{a}}$ \\ a Naval Medical Research Center, Infectious Diseases Directorate, Silver Spring, MD, United States \\ ${ }^{\mathrm{b}}$ Uniformed Services University of the Health Sciences, Department of Preventive Medicine and Biometrics, 4301 Jones Bridge Road, Bethesda, MD, United States
}

\section{A R T I C L E I N F O}

\section{Article history:}

Available online 6 August 2011

\section{Keywords:}

Dengue

DNA vaccines

Clinical trials

Non-human primates

Shuffled DNA vaccines

\begin{abstract}
A B S T R A C T
Vaccination with plasmid DNA against infectious pathogens including dengue is an active area of investigation. By design, DNA vaccines are able to elicit both antibody responses and cellular immune responses capable of mediating long-term protection. Great technical improvements have been made in dengue DNA vaccine constructs and trials are underway to study these in the clinic. The scope of this review is to highlight the rich history of this vaccine platform and the work in dengue DNA vaccines accomplished by scientists at the Naval Medical Research Center. This work resulted in the only dengue DNA vaccine tested in a clinical trial to date. Additional advancements paving the road ahead in dengue DNA vaccine development are also discussed.
\end{abstract}

Published by Elsevier Ltd.

\section{Introduction}

Since the discovery in the 1990s that genetically engineered DNA can be delivered in vaccine form and elicit an immune response, there has been considerable progress in fully understanding the nucleic acid immunization technology. The ability of plasmid DNA to activate both arms of the immune system (i.e. cellular and humoral) against an encoded gene product has resulted in its intensive study for vaccine development, especially for pathogens that likely require a cellular immune response for protection such as malaria and human immunodeficiency virus (HIV).

Observations in the early 1990s that in vitro and in vivo gene transfer of recombinant DNA by a variety of techniques leading to protein expression formed the basis for the first DNA vaccine candidates testing in rodents and non-human primates (NHP). Some of these approaches included retroviral gene transfer, using DNA formulations with liposomes and calcium phosphase-co-precipitated DNA [1,2]. Research done by Wolff et al. [3] showed direct intramuscular inoculation of plasmid DNA encoding several unique reporter genes could induce protein expression. This study provided a strong basis for the delivery of recombinant nucleic acids ("naked DNA") in vivo. Continued successes included the work of Tang et al. who showed that mice injected with plasmid DNA encoding human growth hormone could elicit antigen-specific antibody responses and by Robinson et al. who showed DNA vaccination of chickens against influenza could mediate protective

\footnotetext{
* Corresponding author. Tel.: +1 301319 7320; fax: +1 3013197451.

E-mail address: Janine.danko@med.navy.mil (J.R. Danko).
}

immunity [4,5]. Another influenza DNA vaccine mouse study further showed that both antibody and CD8+ cytotoxic lymphocyte (CTL) responses were elicited, confirming that DNA vaccines stimulate both humoral and cellular immunity [6]. These and many other early studies provided evidence that the DNA vaccine platform was unique, and a technically simple means to induce desired immune responses to protect against infectious diseases [7]. Given the need for an effective dengue vaccine to generate a tetravalent immune response, exploring the use of the DNA vaccine platform was a logical step.

\section{How DNA vaccines work}

There are several factors that influence the type of immune response induced by DNA vaccination. DNA vaccines consist of the optimized gene sequence of interest cloned into a bacterial plasmid. The expression plasmid backbone and the specific nucleotide sequences chosen from the pathogen of interest play a critical role in the immunogenicity. The plasmid DNA is engineered synthetically or by PCR for optimal expression in eukaryotic cells. It is understood that the amount of antigen produced in vivo after DNA inoculation is usually in the picogram to nanogram range. Since small amounts of protein are synthesized, an efficient immune response is dependent on the type of antigen-presenting cells (APCs) transfected and/or the immune-enhancing properties of the DNA itself. Research delineating the cellular mechanisms involved in the processing and presenting of DNA vaccine-induced antigens in vivo is described in detail by Gurunathan et al. [8] and is summarized here.

The optimized gene sequence is cloned into the plasmid backbone and then injected into either the skin, subcutaneum or muscle. 


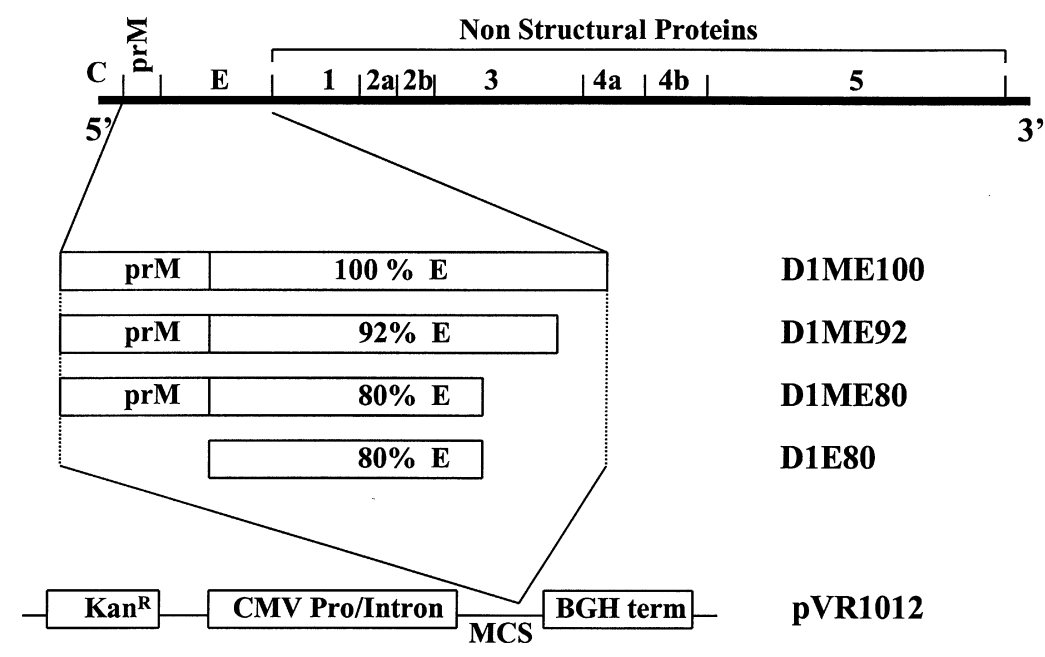

\section{DEN-1 Genome} ,

D1ME100

D1ME92

D1ME80

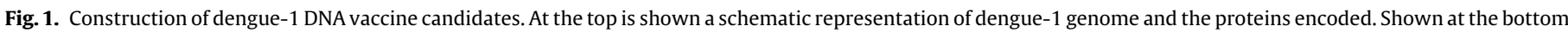

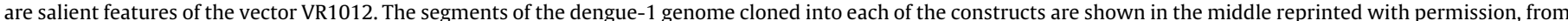
Raviprakash et al. [12].

The plasmid DNA is taken up by APCs and the plasmid-encoded genes expressed intracellularly to generate the target antigens. These host-synthesized antigens can then become the subject of immune surveillance when associated with major histocompatibility complex (MHC) class I and MHC class II proteins of the vaccinated person. Antigen-loaded APCs travel to the draining lymph nodes via the afferent lymphatic vessels where they present peptide antigens via the MHC and the T cell receptors in combination with signaling by co-stimulatory molecules to naïve $\mathrm{T}$ cells. Additionally, shed antigen can be captured by specific high-affinity immunologlobulins (B cell receptors) expressed on the surface of $B$ cells, thus facilitating the induction of a B-cell response. In contrast to traditional vaccines, which are primarily processed via the MHC class II system and lead to stimulation of T-helper lymphocytes, the intracellular expression and processing associated with DNA vaccines allows for presentation to the immune system in the context of MHC class I system, offering the stimulation of specific cytotoxic effector $\mathrm{T}$ cells. The above cellular interactions initiate, activate and expand $\mathrm{T}$ and $\mathrm{B}$ cells, and stimulate both humoral and cellular immune responses.

\section{Dengue DNA vaccine design and pre-clinical evaluation}

\subsection{DNA vaccine design strategies}

The antibody response to dengue in humans is directed at a variety of dengue proteins including C, prM, E, NS1, NS3, NS4B and NS5. The majority of the anti-dengue neutralizing antibody epitopes have been mapped to the E protein. For this reason, scientists at the Naval Medical Research Institute chose the E gene for developing the dengue DNA vaccine construct. Studies show that the prM gene is required for proper processing and folding of the E protein, so the prM gene was also included $[9,10]$. To determine the size of the E gene to include, four different dengue- 1 constructs were produced using the VR1012 plasmid backbone and the Western Pacific 74 strain. Two of the constructs contained E genes lacking the carboxy terminal membrane anchor sequences to facilitate secretion of the E protein translated from the DNA vaccine. In one construct, $20 \%$ of the E gene carboxy terminus was deleted (prM80) and in the other, $8 \%$ was deleted (prM92) (Fig. 1). The $80 \%$ E gene without prM was included as a candidate based on the results of Men et al. [11], which showed that vaccinia constructs expressing greater than $79 \% \mathrm{E}$ protein were immunogenic and those express- ing proteins between 59\% and 81\% E were secreted extracellularly. Smaller and larger expressed proteins were retained intracellularly.

Small animal studies were conducted to evaluate the various DNA vaccine constructs. The constructs containing $80 \%$ of the $\mathrm{E}$ gene (E80) and $100 \%$ of the E gene including the prM gene (ME100), elicited the best neutralizing antibody responses in mice. The latter DNA vaccine construct produced a more sustained neutralizing antibody response compared to the E80 candidate [12]. Further evaluation of the in vitro expression of ME100 revealed the production of virus-like particles. Based on these data, we chose to include the prM gene along with $100 \%$ of the E gene as the platform for developing a tetravalent dengue DNA vaccine. The combination of four different plasmids, each expressing prM and $\mathrm{E}$ proteins of a different serotype, constitutes the tetravalent vaccine product. The Western Pacific 74 strain was used for the dengue-1 construct and low passage Philippine strains were used for the dengue-2, -3 and -4 constructs.

Raviprakash and others performed parallel studies to evaluate the use of shuffled DNA technology to develop a single DNA vaccine plasmid construct capable of producing tetravalent neutralizing antibody responses. Three candidate shuffled DNA vaccines generated using this technology elicited tetravalent neutralizing antibodies in mice and protected against a lethal dengue- 2 (New Guinea C strain) live virus challenge [13]. However, upon testing in a non-human primate (NHP) model, only two of the three shuffled vaccine candidates gave rise to tetravalent neutralizing antibodies along with the tetravalent wild-type control that consisted of a mixture of plasmids representing each serotype [14]. Upon challenge with live dengue-1 (WestPac) and dengue-2 viruses, only partial protection against dengue- 1 was observed and despite evidence of priming, none of the animals were protected against dengue- 2 . For the dengue- 1 challenge, the tetravalent mixture and one shuffled vaccine candidate produced the fewest mean days of dengue-1 viremia post-challenge. Despite observing no protection against the dengue- 2 live virus challenge, the best neutralizing antibody response occurred with the control tetravalent mixture.

To improve the immunogenicity of dengue DNA vaccines, investigators performed studies utilizing endosomal/lysosomal targeting of antigens expressed by the vaccine. Targeting antigens to the endomsomal/lysosomal compartment results in the colocalization of the expressed dengue protein with MHC class II. This co-localization with MHC class II molecules presumably enhances MHC class II presentation of antigens and neutralizing antibody 
responses. To accomplish this, the transmembrane and cytoplasmic regions of the $\mathrm{E}$ gene were replaced with sequences that express the lysosome-associated membrane protein (LAMP). Initial studies were conducted using the dengue- $2 \mathrm{E}$ gene. Mice immunized with the prME-LAMP chimera demonstrated a significantly higher antidengue-2 neutralizing response compared to animals immunized with the unmodified prME DNA vaccine construct [14,15]. Attempts to engineer dengue-LAMP chimeras for the other serotypes resulted in constructs that failed to track appropriately to the endosomal/lysosomal compartment (unpublished results).

Based on the studies described above, we chose to take forward for further non-human primate testing, the tetravalent dengue DNA vaccine consisting of DNA plasmids for each serotype. Given the improved neutralizing antibody responses seen in mice, researchers chose the dengue 2 prME-LAMP chimera for the dengue-2 component of the tetravalent dengue DNA vaccine.

\subsection{Pre-clinical non-human primate studies}

Although no perfect animal models of human dengue disease exist, NHPs can be used to evaluate immunogenicity and allow immunization schedules that are more aligned to the frequency anticipated in humans. Virus neutralizing antibodies are readily detected in several NHP species and viremia can be measured postchallenge as a surrogate of protection.

Three studies with the dengue 1 monovalent DNA vaccine (D1ME100) provided the basis for advancement into the clinic. In the first study [16], eight rhesus macaques (Macaca mulatta) received $1.0 \mathrm{mg}$ of plasmid DNA intramuscularly (IM) in phosphatebuffered saline (PBS) at months 0,1 and 5. Control animals $(n=3)$ received plasmid without dengue genes. All vaccinated animals seroconverted by ELISA, and neutralizing antibodies were produced in 7 of 8 vaccinated NHPs and none of the controls. These animals were challenged with wild type dengue-1 (strain WestPac 74) 4 months after the third dose of vaccine ( 9 months after first dose). After challenge, 4 of 8 vaccinated animals were completely protected from viremia and the remaining four had reduced viremia. T-cell stimulation was examined by stimulating peripheral blood lymphocytes from two naïve primates and two vaccinated primates and measured by in vitro secretion of IFN-gamma by ELISA. By this method, T-cell responses were undetectable in the vaccinated animals prior to the live virus challenge. Because the ELISA method was less sensitive than the ELISpot and intracellular cytokine assays (not available at that time), low level memory anti-dengue T-cell responses may have gone undetected. After live virus challenge, robust anti-dengue-1 $\mathrm{T}$-cell responses were demonstrated.

The second study evaluated the D1ME100 vaccine in New World Owl monkeys (Aotus nancymae) [17]. Six animals received $1.0 \mathrm{mg}$ of plasmid DNA (three IM and three ID) at months 0,1 and 5 . Control monkeys $(n=3)$ received vector plasmid alone. All six vaccinated animals produced neutralizing antibodies. Monkeys were challenged with wild type dengue- 1 virus at 6 months after the third vaccine dose (11 months after first dose). After challenge, 2 of 6 animals were completely protected from viremia and the remainder had reduced viremia. These studies with initial formulations were a successful test of concept that dengue DNA vaccines can protect NHPs from challenge with wild type virus.

A third NHP study was conducted in Aotus monkeys to enhance neutralizing antibody responses to the D1ME100 vaccine [18]. Four strategies were evaluated: (1) co-immunization with a plasmid expressing Aotus GM-CSF gene, (2) co-immunization with a plasmid containing human immunostimulatory sequences (ISS), (3) co-immunization with both the GM-CSF gene and ISS, and (4) delivery of vaccine using the needle-free Biojector ${ }^{\circledR}$ system. Immunization with the various vaccine formulations, either by needle injection or Biojector ${ }^{\circledR}$, led to neutralizing antibody $\left(\mathrm{PRNT}_{50}\right.$ group means ranging from $1: 156.2$ to $1: 357.5)$ that was stable for up to 6 months after vaccination. Furthermore, 6 of 7 monkeys (85\%), and 7 of 8 monkeys ( $87 \%$ ) receiving this formulation were completely protected from viremia when challenged with a WestPac dengue- 1 strain, 1 and 6 months after vaccination, respectively. Neutralizing antibody levels were higher in the group that was immunized using Biojector $^{\circledR}$ compared to levels obtained using needle and syringe (anti-dengue-1 PRNT $_{50}$ group mean, 1:307.8 versus $1: 161.8$ ). This was a significant improvement compared to our previous studies with D1ME100, thus it was the first dengue DNA vaccine selected for evaluation in humans.

\section{Dengue DNA vaccine clinical trials}

The accomplishments of the early animal studies and the advancement of plasmid DNA technology led to the first human clinical trial of DNA vaccination against HIV-1 infection in 1998 [19]. Since then, other DNA vaccines for infectious diseases have been evaluated in published Phase 1 studies including other HIV-1 constructs, an Ebola virus candidate $[20,21]$ as well as a West Nile virus (WNV) vaccine [22]. The West Nile vaccine candidate encoded the prM and E glycoproteins identical to that in the WNV isolate from the epidemic experienced in the U.S. in 1999. The vaccines were well-tolerated and safe. All subjects receiving three doses mounted a neutralizing antibody response $\left(\mathrm{PRNT}_{50}\right.$ peak $\left.=1: 128\right)$, however when measured using the more stringent PRNT 75 , there were minimal responses (peak=1:32). When WNV reporter-virus particles were used to measure antibody-mediated neutralization using a Raji B lymphoblastoid (surrogate) cell line that expressed the WNV attachment factor CD209L (DC-SIGNR), the neutralizing antibody titers were magnified. Vaccine-specific T-cell responses specific for WNV insert-specific peptide pools as measured by ELISpot assays showed the majority of vaccinees mounted both CD4+ and CD8+ T-cell responses.

To date, our Phase 1 study is the only published dengue DNA vaccine human clinical trial [23]. The dengue-1 D1ME100 vaccine construct evaluated in the trial was similar to the WNV DNA vaccine described above (DNA plasmid expressing prM and E) and was also administered intramuscularly using Biojector ${ }^{\circledR}$.

To evaluate the pre-clinical safety of D1ME100 prior to beginning the clinical trial, a 61-day single-dose IM biodistribution study was performed in CD-1 mice (Gene Logic Laboratories Inc., Study No. 1381-101). Two groups of CD-1 mice (33 mice total) were given either $50 \mu \mathrm{l}$ of sterile PBS (10 mice) or $100 \mu \mathrm{g}$ in $50 \mu \mathrm{l}$ PBS (23 mice) of D1ME100 IM. No significant changes were noted among the groups with regard to mortality, clinical signs of toxicity, body weights, body weight gains, or food consumption. Distribution studies showed the presence of D1ME100 in the injection site muscle at the day 62 necropsy, but showed a progressive decrease in copy number per microgram of genomic DNA between necropsies.

Integration of DNA from the vaccine into host chromosomal DNA resulting in neoplastic transformation of cells has been a theoretical risk. In the above bio-distribution studies of D1ME100, no evidence of integration was observed through study day 62 in CD-1 mice. Examination of in vivo genomic DNA integration of plasmid DNA sequences (unrelated to the NMRC vaccine) into host genomic DNA was conducted in mice by Martin et al. [24]. Using quantitative PCR amplification, integration of plasmid DNA was estimated to be a low probability event, occurring 3000 times lower than the spontaneous mutation rate. Subsequently, Wang et al. [25] demonstrated negligible uptake occurs in mice when DNA is administered via electroporation. To date, clinical Phase 1 human trials of DNA vaccines (including D1ME100) have not demonstrated the occurrence of either integration or neoplastic transformation in human recipients. 


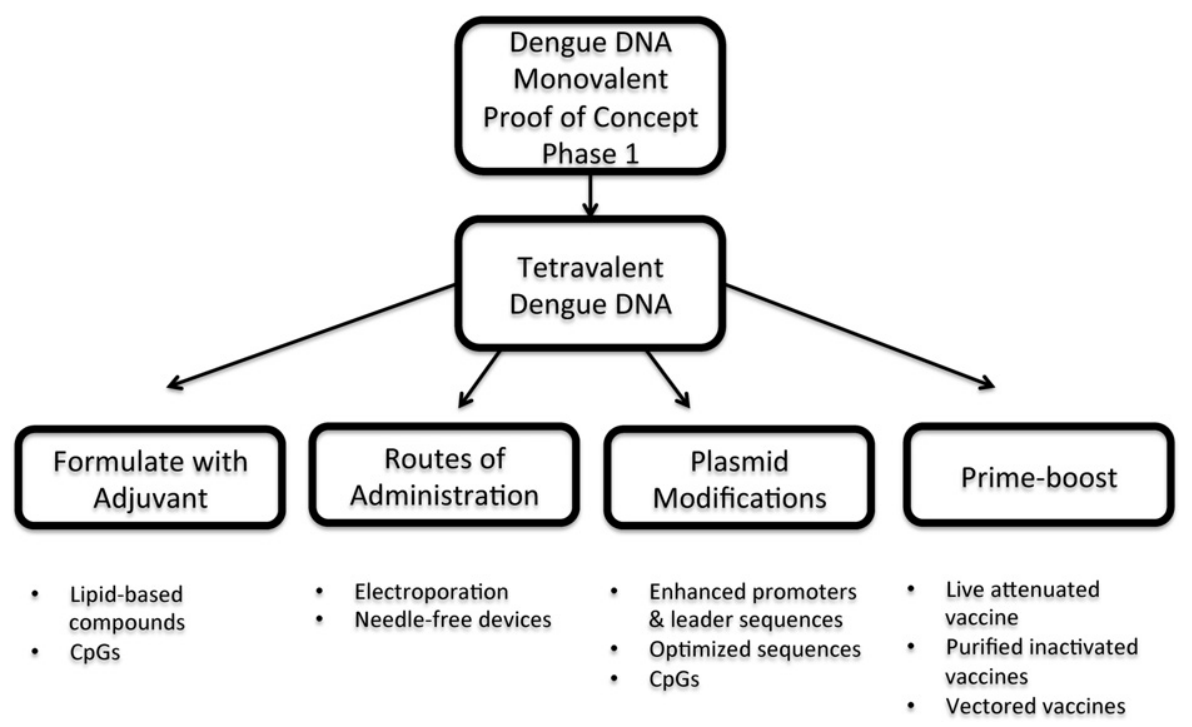

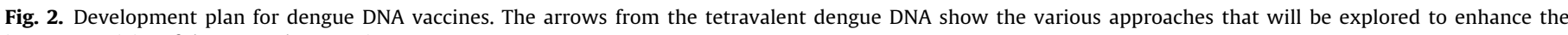
immunogenicity of the tetravalent vaccine.

A toxicity study was performed in New Zealand white rabbits (Gene Logic Laboratories Inc., Study No. 1381-102). The study was designed as a 14-week repeat-dose, where groups of rabbits ( 8 animals/sex/group) were used with one group receiving $2.5 \mathrm{mg}$ of the vaccine IM, the other receiving $5.0 \mathrm{mg} \mathrm{IM}$, and the control group receiving sterile PBS. Injections were repeated on days 29,57 , and 85 . No significant changes were noted among the groups in mortality, cage side observations, clinical observations, Draize observations, body weights, body weight changes, food consumption, ophthalmologic, clinical pathology, bone marrow, or immunologic assessments, organ weights, organ rations, gross pathologic, or histopathologic observations. Under these study conditions, repeated IM administration of doses of up to $5.0 \mathrm{mg}$ of D1ME100 to New Zealand White rabbits also failed to show any overt systemic or local toxicity.

For the Phase 1 clinical trial, D1ME100 DNA vaccine doses of $1.0 \mathrm{mg} / \mathrm{ml}$ and $5.0 \mathrm{mg} / \mathrm{ml}$ were studied in healthy flavivirus-naïve subjects administered at 0,1 and 5 months. Anti-dengue 1 plaque reduction neutralization titers $\left(\mathrm{PRNT}_{50}\right)$ ranged from $1: 11$ to $1: 135$, with a median maximum titer for the five responsive subjects in the high dose group of 1:50. No neutralizing antibody responses were detectable in subjects who received the low dose vaccine. To evaluate cell-mediated immunity, IFN- $\gamma$ levels were assessed against $\mathrm{E}$ proteins from all four dengue virus serotypes. Responses were detected in $50 \%(4 / 8)$ and $83.3 \%(10 / 12)$ of subjects in the low and high dose group, respectively.

The vaccine was well-tolerated without any severe vaccinerelated adverse events reported. Mild pain or tenderness and swelling were the most commonly reported symptoms postvaccination. Despite the low $\mathrm{PRNT}_{50}$ titers, overall immune responses and excellent safety profile provided evidence that a dengue DNA vaccine platform is a viable alternative to other vaccines.

\section{The future for dengue DNA vaccine development}

To date, the immunogenicity of DNA vaccines has not been comparable to vaccines developed using more conventional methodologies such as purified inactivated vaccines, live attenuated vaccines and recombinant protein vaccines. The ease of manufacture and stability of DNA vaccines continue to make this development approach attractive, but to be a viable option, the immunogenicity must be improved. Fig. 2 outlines the development plan for dengue DNA vaccines. Enhancements can be made either by improving the DNA construct itself, co-immunization with adjuvants, and/or finding alternate routes of administration that promote more efficient vaccine uptake by antigen-presenting cells and greater expression of encoded proteins.

Improvements in the plasmid backbone of DNA vaccines include the use of different promoters, the inclusion of immunostimulatory sequences, and more efficient signal sequences. In addition to plasmid backbone alterations, genes that express the desired antigen are routinely codon-optimized for better expression in humans. Early pre-clinical small animal and non-human primate studies showed the benefit of including immune-enhancing CpG sequences [26] and tissue plasminogen activator leader sequences in DNA immunization [27]. However, these modified vaccines failed to show adequate immunogenicity when evaluated in human clinical trials [28,29]. CpG motifs continue to be explored for their immune modulating characteristics [30].

Ledgerwood et al., recently published the results of a Phase 1 study of a WNV DNA vaccine containing a promoter that was modified to include regulatory sequences from the long terminal repeat segment of HTLV-I [31]. These sequences enhance transcription and posttranscriptional events. This DNA vaccine modification resulted in significant increases in $\mathrm{T}$ cell responses to the prM protein and in antigen-specific CD $8 \mathrm{~T}$ cell responses to the $\mathrm{E}$ protein compared to the unmodified WNV DNA vaccine. Phase 2 studies to evaluate protection against disease are underway.

DNA vaccine efforts targeting cancer demonstrated the utility of co-administering the vaccine with lipid-based compounds. Targeting infectious disease agents, this approach resulted in enhanced immune responses to influenza, tuberculosis, HIV, anthrax and Japanese Encephalitis. Vaxfectin ${ }^{\circledR}$, a proprietary lipid adjuvant manufactured by Vical Inc. has been studied with DNA vaccines for several infectious pathogens. A Phase 1 human clinical trial of a Vaxectin ${ }^{\circledR}$-formulated H5N1 DNA vaccine showed that the vaccine was well tolerated and induced neutralizing antibody responses comparable to protein-based H5N1 vaccines [32]. No comparison was made to the DNA vaccine administered without Vaxfectin ${ }^{\circledR}$.

With the less than optimal neutralizing antibody response to the prototype dengue-1 DNA vaccine (D1ME100) in humans, we sought to enhance the immunogenicity of vaccine by formulating it with Vaxfectin ${ }^{\circledR}$. Pre-clinical non-human primate studies using 
a Vaxfectin ${ }^{\circledR}$-formulated tetravalent dengue DNA vaccine demonstrated an enhanced anti-dengue neutralizing antibody response against each of the four dengue virus serotypes (unpublished results) compared to the unformulated vaccine. Based on these results, efforts are underway to evaluate the formulated tetravalent vaccine in a Phase 1 clinical trial.

The route of administration may have a significant impact on the immunogenicity of a particular DNA vaccine. Most of the pre-clinical and clinical studies of DNA vaccines involved IM administration by needle or the needle-free Biojector ${ }^{\circledR}$ device. Intradermal administration has also been used with variable success. Recently, electroporation has received attention as a route of administration for improved immunogenicity [33]. The question remains though whether this method of vaccine delivery is conducive for large public health immunization campaigns. Many resource-poor areas would likely have difficulties with any vaccine that relies on an expensive technically advanced delivery system.

The testing of combination vaccines delivered together or in a heterologous prime-boost regimen has recently been studied for many infectious pathogens including dengue. Scientists at NMRC have maintained close collaborations with U.S. Army researchers at the Walter Reed Army Institute of Research (WRAIR) for years. Mouse and NHP studies including the WRAIR purified inactivated dengue (PIV) vaccine in combination with the NMRC DNA dengue vaccine have shown promising results [34,35]. These have led to the planning and regulatory preparations for human trials using prime boost strategies incorporating the dengue DNA, PIV and WRAIR live-attenuated vaccine candidates.

\section{Conclusion}

The field of DNA vaccination has advanced significantly during the last two decades. Better-designed constructs and promoters and novel delivery technologies tested in animals and advanced in the clinic are evidence that the field is exciting. Our upcoming tetravalent dengue DNA vaccine formulated with Vaxfectin ${ }^{\circledR}$ Phase 1 trial and the trials studying prime-boost approaches with DNA candidates will be pivotal in determining if this platform will provide realistic possibilities for a dengue vaccine in the future.

\section{Acknowledgements}

The authors wish to thank Dr. Kanakatte Raviprakash for his kind assistance with editing and review of the manuscript. The views expressed in this article are those of the authors and do not necessarily reflect the official policy or position of the Department of the Navy, Department of Defense or the U.S. Government. The authors are military service members and this work was prepared as part of their official duties. Title 17 U.S.C 105 provides that "Copyright protection under this title is not available for any work of the U.S. Government." Title 17 U.S.C 101 defines a Government work as a work prepared by military service members as part of that person's official duties.

Conflict of interest statement: Kevin Porter is a co-patent holder on US Patent No. US 6,455,509 B1, Title "Dengue Nucleic Acid Vaccines that Induce Neutralizing Antibodies". Charmagne Beckett and Janine Danko: these two authors declare that there is no conflict of interest. Funding: This work described in this manuscript was supported and funded by the Military Infectious Diseases Research Program and NMRC work unit Flavivirus Vaccine Research 62787A.870.S.A0014 and A0235.

\section{References}

[1] Mannino RJ, Gould-Fogerite S. Liposome mediated gene transfer. Biotechniques 1988;6(July-August (7)):682-90.
[2] Nicolau C, Le Pape A, Soriano P, Fargette F, Juhel MF. In vivo expression of rat insulin after intravenous administration of the liposome-entrapped gene for rat insulin I. Proc Natl Acad Sci USA 1983 Feb;80(February (4)):1068-72.

[3] Wolff JA, Malone RW, Williams P, Chong W, Acsadi G, Jani A, et al. Direct gene transfer into mouse muscle in vivo. Science 1990;247(March (4949 Pt 1)):1465-8.

[4] Robinson HL, Hunt LA, Webster RG. Protection against a lethal influenza virus challenge by immunization with a haemagglutinin-expressing plasmid DNA. Vaccine 1993;11(9):957-60.

[5] Tang DC, De Vit M, Johnston SA. Genetic immunization is a simple method for eliciting an immune response. Nature 1992;356(6365):152-4.

[6] Ulmer JB, Donnelly JJ, Parker SE, Rhodes GH, Felgner PL, Dwarki VJ, et al. Heterologous protection against influenza by injection of DNA encoding a viral protein. Science 1993;259(5102):1745-9.

[7] Kutzler MA, Weiner DB. DNA vaccines: ready for prime time. Nat Rev Genet 2008;9(October (10)):776-88.

[8] Gurunathan S, Klinman DM, Seder RA. DNA vaccines: immunology, application, and optimization. Annu Rev Immunol 2000;18:927-74.

[9] Lorenz IC, Allison SL, Heinz FX, Helenius A. Folding and dimerization of tickborne encephalitis virus envelope proteins prM and $\mathrm{E}$ in the endoplasmic reticulum. J Virol 2002;76(June (11)):5480-91.

[10] Mukhopadhyay S, Kuhn RJ, Rossmann MG. A structural perspective of the flavivirus life cycle. Nat Rev 2005;3:13-22.

[11] Men RH, Bray M, Lai CJ. Carboxy-terminally truncated dengue virus envelope glycoproteins expressed on the cell surface and secreted extracellularly exhibit increased immunogenicity in mice. J Virol 1991;65(3):1400-7. ISSN:0022538x.

[12] Raviprakash K, Kochel TJ, Ewing D, Simmons M, Phillips I, Hayes CG, et al. Immunogenicity of dengue virus type 1 DNA vaccines expressing truncated and full length envelope protein. Vaccine 2000;18(22):2426-34.

[13] Apt D, Raviprakash K, Brinkman A, Semyonov A, Yang S, Skinner C, et al. Tetravalent neutralizing antibody response against four dengue serotypes by a single chimeric dengue envelope antigen. Vaccine 2006;24(January (3)): 335-44.

[14] Raviprakash K, Apt D, Brinkman A, Skinner C, Yang S, Dawes G, et al. A chimeric tetravalent dengue DNA vaccine elicits neutralizing antibody to all four virus serotypes in Rhesus macaques. Virology 2006;353(September (1)):166-73.

[15] Lu Y, Raviprakash K, Leao IC, Chikhlikar PR, Ewing D, Anwar A, et al. Dengue 2 PreM-E/LAMP chimera targeted to the MHC class II compartment elicits longlasting neutralizing antibodies. Vaccine 2003;21(May (17-18)):2178-89.

[16] Raviprakash K, Porter KR, Kochel TJ, Ewing D, Simmons M, Phillips I, et al. Dengue virus type 1 DNA vaccine induces protective immune responses in rhesus macaques. J Gen Virol 2000;81(July (Pt 7)):1659-67.

[17] Kochel TJ, Raviprakash K, Hayes CG, Watts DM, Russell KL, Gozalo AS, et al. A dengue virus serotype-1 DNA vaccine induces virus neutralizing antibodies and provides protection from viral challenge in Aotus monkeys. Vaccine 2000;18(July (27)):3166-73.

[18] Raviprakash K, Ewing D, Simmons M, Porter KR, Jones TR, Hayes CG, et al. Needle-free Biojector injection of a dengue virus type 1 DNA vaccine with human immunostimulatory sequences and the GM-CSF gene increases immunogenicity and protection from virus challenge in Aotus monkeys. Virology 2003;315(October (2)):345-52.

[19] MacGregor RR, Boyer JD, Ugen KE, Lacy KE, Gluckman SJ, Bagarazzi ML, et al. First human trial of a DNA-based vaccine for treatment of human immunodeficiency virus type 1 infection: safety and host response. J Infect Dis 1998;178(July (1)):92-100.

[20] Graham BS, Koup RA, Roederer M, Bailer RT, Enama ME, Moodie Z, et al. Phase 1 safety and immunogenicity evaluation of a multiclade HIV-1 DNA candidate vaccine. J Infect Dis 2006;194(December (12)):1650-60.

[21] Martin JE, Sullivan NJ, Enama ME, Gordon IJ, Roederer M, Koup RA, et al. A DNA vaccine for Ebola virus is safe and immunogenic in a phase I clinical trial. Clin Vaccine Immunol 2006;13(November (11)):1267-77.

[22] Martin JE, Pierson TC, Hubka S, Rucker S, Gordon IJ, Enama ME, et al. A West Nile virus DNA vaccine induces neutralizing antibody in healthy adults during a phase 1 clinical trial. J Infect Dis 2007;196(December (12)):1732-40.

[23] Beckett CG, Tjaden J, Burgess T, Danko JR, Tamminga C, Simmons M, et al. Evaluation of a prototype dengue-1 DNA vaccine in a Phase 1 clinical trial. Vaccine $2011 ; 29($ January (5)):960-8.

[24] Martin T, Parker SE, Hedstrom R, Le T, Hoffman SL, Norman J. et al. Plasmid DNA malaria vaccine: the potential for genomic integration after intramuscular injection. Hum Gene Ther 1999;10(March (5)):759-68.

[25] Wang Z, Troilo PJ, Wang X, Griffiths TG, Pacchione SJ, Barnum AB, et al. Detection of integration of plasmid DNA into host genomic DNA following intramuscular injection and electroporation. England: Gene Ther; 2004. pp. 711-21.

[26] Porter KR, Kochel TJ, Wu SJ, Raviprakash K, Phillips I, Hayes CG. Protective efficacy of a dengue 2 DNA vaccine in mice and the effect of CpG immunostimulatory motifs on antibody responses. Arch Virol 1998;143(5):997-1003.

[27] Gramzinski RA, Maris DC, Doolan D, Charoenvit Y, Obaldia N, Rossan R, et al. Malaria DNA vaccines in Aotus monkeys. Vaccine 1997;15(8):913-5.

[28] Le TP, Coonan KM, Hedstrom RC, Charoenvit Y, Sedegah M, Epstein JE, et al. Safety, tolerability and humoral immune responses after intramuscular administration of a malaria DNA vaccine to healthy adult volunteers. Vaccine 2000;18(18):1893-901.

[29] Wang R, Doolan DL, Le TP, Hedstrom RC, Coonan KM, Charoenvit Y, et al. Induction of antigen-specific cytotoxic T lymphocytes in humans by a malaria DNA vaccine. Science 1998;282(5388):476-80. 
[30] Bode C, Zhao G, Steinhagen F, Kinjo T, Klinman DM. CpG DNA as a vaccine adjuvant. Expert Rev Vaccines 2011;10(April (4)):499-511.

[31] Ledgerwood JE, Pierson TC, Hubka SA, Desai N, Rucker S, Gordon IJ, et al. A West Nile virus DNA vaccine utilizing a modified promoter induces neutralizing antibody in younger and older healthy adults in a phase I clinical trial. J Infect Dis 2011;10(May (203)):1396-404.

[32] Smith LR, Wloch MK, Ye M, Reyes LR, Boutsaboualoy S, Dunne CE, et al. Phase 1 clinical trials of the safety and immunogenicity of adjuvanted plasmid DNA vaccines encoding influenza A virus H5 hemagglutinin. Vaccine 2010;28(March (13)):2565-72
[33] Barbon CM, Baker L, Lajoie C, Ramstedt U, Hedley ML, Luby TM. In vivo electroporation enhances the potency of poly-lactide co-glycolide (PLG) plasmid DNA immunization. Vaccine 2010;28(November (50)):7852-64.

[34] Simmons M, Burgess T, Lynch J, Putnak R. Protection against dengue virus by non-replicating and live attenuated vaccines used together in a prime boost vaccination strategy. Virology 2010:280-8.

[35] Simmons M, Porter KR, Hayes CG, Vaughn DW, Putnak R. Characterization of antibody responses to combinations of a dengue virus type 2 DNA vaccine and two dengue virus type 2 protein vaccines in rhesus macaques. J Virol 2006;80(October (19)):9577-85. 